PHYSICAL REVIEW D 82, 127101 (2010)

\title{
Remarks on thermoelastic effects at low temperatures and quantum limits in displacement measurements
}

\author{
Kentaro Somiya* \\ Waseda Institute for Advanced Study, 1-6-1 Nishiwaseda, Shinjuku, Tokyo 169-8050, Japan
}

Keiko Kokeyama

School of Physics and Astronomy, University of Birmingham, Edgbaston, Birmingham B15 2TT, United Kingdom

Ronny Nawrodt

Institut für Festkörperphysik, Friedrich-Schiller-Universität Jena, D-07743 Jena, Germany

(Received 31 August 2010; published 1 December 2010)

\begin{abstract}
The noise level of thermoelastic fluctuations of a mirror at low temperature was derived and introduced by M. Cerdonio et al. [Phys. Rev. D 63, 082003 (2001)]. The importance of the paper has become remarkable according to the increasing attention to the low-temperature high-precision measurement device. Here we would like to introduce the analytical form of their equation of thermoelastic noise in the integral form in order to reduce the computational labor.
\end{abstract}

DOI: 10.1103/PhysRevD.82.127101

PACS numbers: 04.80.Nn, 05.40.-a, 42.50.Lc

\section{INTRODUCTION}

The first-generation gravitational-wave detectors such as LIGO, Virgo, and GEO have reached their designed goal sensitivity and the second-generation detectors such as Advanced LIGO, Advanced Virgo, GEO HF, and LCGT are in the construction phase. A third-generation detector ET is also planned and the design study is underway. Among those, LCGT and ET will be operated in the cryogenic temperature, accommodating mirrors and suspension fibers made of sapphire (LCGT) or silicon (ET) $[1,2]$.

Those materials with high thermal conductivity are suitable for the low-temperature operation, but at the same time thermoelastic noise is going to be an issue. Thermoelastic noise, first introduced by Braginsky et al. [3], is caused by temperature fluctuation that is converted to the volume fluctuation via thermal expansion. Thus, at least at room temperature the thermoelastic noise of the potential materials for cryogenic operation is high. Silicon shows two special temperatures, 18 and $125 \mathrm{~K}$, where the coefficient of thermal expansion vanishes and therefore the thermoelastic noise is zero as well [4].

Let us first review the equations for thermoelastic noise introduced by Cerdonio et al. [5]. We will then introduce our equation that allows a much quicker computation.

\section{THERMOELASTIC NOISE}

The power spectrum of thermoelastic noise at room temperature is given by Braginsky et al. [3] and is also introduced in Ref. [5] as the noise level in the adiabatic limit:

*somiya@aoni.waseda.jp

$$
\begin{aligned}
S_{x}(\omega) & =\frac{16 k_{\mathrm{B}} T^{2}\left(1+\nu_{s}\right)^{2} \kappa_{s} \alpha^{2}}{\sqrt{\pi} C_{s}^{2} w_{0}^{3} \omega^{2}} \quad\left[\omega_{c} \ll \omega\right], \\
\omega_{c} & =\frac{2 \kappa_{s}}{C_{s} w_{0}^{2}} .
\end{aligned}
$$

Here $k_{\mathrm{B}}$ is the Boltzmann's constant, $T$ is temperature, $\nu_{s}$ is Poisson's ratio, $\alpha_{s}$ is thermal expansion, $C_{s}$ is specific heat per volume, $w_{0}$ is the beam radius on the mirror, and $\omega$ is the angular frequency of the measurement.

The analytical expression of thermoelastic noise that fits well at low temperature is introduced by Yamamoto for the study of LCGT [6]:

$$
S_{x}(\omega)=\frac{2 k_{\mathrm{B}} T^{2}\left(1+\nu_{s}\right)^{2} \alpha^{2}}{\sqrt{\pi \kappa_{s} C_{s} \omega}} \quad\left[\omega \ll \omega_{c}\right] .
$$

The relaxation time is short at low temperature and the noise level does not depend on the beam radius.

Equation (1) is good for room temperature and/or at high frequencies while Eq. (2) is good for low temperature and/or at low frequencies. Cerdonio et al. has derived an equation that is good for arbitrary temperature at any frequencies [Eqs. (20) and (21) in Ref. [5]]:

$$
S_{x}(\omega)=\frac{4 k_{\mathrm{B}} T^{2} \alpha^{2}\left(1+\nu_{s}\right)^{2} w_{0}}{\sqrt{\pi} \kappa_{s}} J\left(\Omega_{c}\right)
$$

$J\left(\Omega_{c}\right)=\sqrt{\frac{2}{\pi^{3}}} \int_{0}^{\infty} \int_{-\infty}^{\infty} \frac{u^{3} e^{-u^{2} / 2}}{\left(u^{2}+v^{2}\right)\left[\left(u^{2}+v^{2}\right)^{2}+\Omega_{c}^{2}\right]} d v d u$, 
with $\Omega_{c}=\omega / \omega_{c}$ as a dimensionless frequency. Equation (21) in Ref. [5] has $\sqrt{2 / \pi}$ instead of $\sqrt{2 / \pi^{3}}$ in front of the integral, which is a typo and has been corrected here. Comparing Eqs. (1)-(3), we can see that $J(\Omega)$ takes the following forms in the high-temperature and lowtemperature limits:

$$
\begin{gathered}
J\left(\Omega_{c}\right) \rightarrow J_{\mathrm{H}}\left(\Omega_{c}\right)=1 / \Omega_{c}^{2} \quad\left[1 \ll \Omega_{c}\right], \\
J\left(\Omega_{c}\right) \rightarrow J_{\mathrm{L}}\left(\Omega_{c}\right)=1 / \sqrt{8 \Omega_{c}} \quad\left[\Omega_{c} \ll 1\right] .
\end{gathered}
$$

In fact, Eq. (2) was introduced referring to the exact solution by Cerdonio et al. by resolving the integral part of Eq. (4) and fitting the coefficients.

\section{ANALYTICAL FORM OF THERMOELASTIC-NOISE EQUATION}

While Eq. (3) lets us calculate thermoelastic noise for arbitrary temperatures, it takes time to actually compute $J\left(\Omega_{c}\right)$ numerically. One may sometimes want to know the temperature dependence of thermoelastic noise. Since the parameters $\kappa_{s}, C_{s}$, and $\alpha_{s}$ have strong dependence on the temperature, $\Omega_{c}$ varies for different temperature. This will additionally increase the computational time needed to evaluate the integral. Instead it would be useful to have the analytical form of Eq. (3) for such cases.

What we need is just some sequence of algebra to resolve $J\left(\Omega_{c}\right)$ into the analytical form without the integral. Using the residue theorem, the integral over $v$ can be resolved. The function $J\left(\Omega_{c}\right)$ reads

$$
\begin{aligned}
J\left(\Omega_{c}\right)= & \sqrt{\frac{2}{\pi}} \int_{0}^{\infty} \frac{u^{2} e^{-u^{2} / 2}}{\Omega_{c}^{2}} \\
& \times\left[1-\frac{u}{\sqrt{2}} \sqrt{\frac{u^{2}+\sqrt{u^{4}+\Omega_{c}^{2}}}{u^{4}+\Omega_{c}^{2}}}\right] d u .
\end{aligned}
$$

This can be finally resolved into the following form with the Gauss error function:

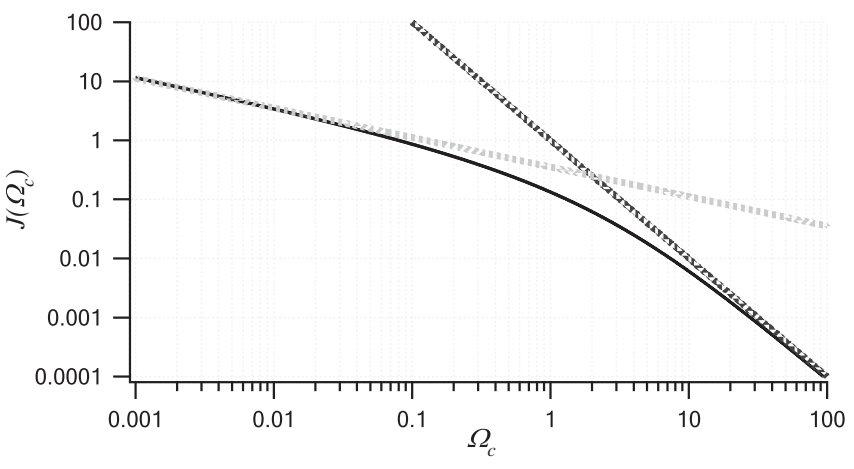

FIG. 1. Exact values of the function $J\left(\Omega_{c}\right)$ and its approximated values at low temperature and high temperature.

$$
\begin{aligned}
J\left(\Omega_{c}\right)= & \operatorname{Re}\left[\frac{e^{i \Omega_{c} / 2}\left(1-i \Omega_{c}\right)}{\Omega_{c}^{2}}\left(\operatorname{Erf}\left[\frac{\sqrt{\Omega_{c}}(1+i)}{2}\right]-1\right)\right] \\
& +\frac{1}{\Omega_{c}^{2}}-\sqrt{\frac{1}{\pi \Omega_{c}^{3}}} .
\end{aligned}
$$

One should just plug this into Eq. (3) to get the power spectrum of thermoelastic noise. Figure 1 shows that $J\left(\Omega_{c}\right)$ in the exact form (8) coincides with the approximated ones in the low-temperature extreme and in the hightemperature extreme.

The importance of the work done by Cerdonio et al. is remarkable not only for the gravitational-wave detectors but also for other experiments in which sensitivity can be limited by thermal noise. The analytical form given in this paper will be useful for a quick evaluation of thermoelastic noise for various experiments.

\section{ACKNOWLEDGMENTS}

We would like to thank Dr. Kazuhiro Yamamoto for valuable discussions on this issue. R. N. acknowledges the support of the German science foundation under contract SFB Transregio 7, and K. K. acknowledges the support of the Science and Technology Facilities Council and the European Commission (FP7 Grant Agreement 211743).
[1] K. Kuroda et al., Int. J. Mod. Phys. D 8, 557 (1999).

[2] M. Punturo et al., Classical Quantum Gravity 27, 084007 (2010).

[3] V. B. Braginsky, M. L. Gorodetsky, and S. P. Vyatchanin, Phys. Lett. A 264, 1 (1999).
[4] Properties of Crystalline Silicon, edited by R. Hull (INSPEC, The Institution of Electrical Engineers, London, United Kingdom, 1999).

[5] M. Cerdonio, L. Conti, A. Heidmann, and M. Pinard, Phys. Rev. D 63, 082003 (2001).

[6] K. Yamamoto, JGW-T0400030-v4, Sec. 3, 2009. 